\title{
Congenital Tuberculosis
}

\author{
Lt Col RPS Tomar*, Brig A Gupta ${ }^{+}$, Brig TR Prasad", Col PJS Bhalla**, Col GSN Murthy (Retd) ${ }^{++}$
}

MJAFI 2008; 64 : 78-80

Key Words: Congenital tuberculosis

\section{Introduction}

$\mathrm{T}$ uberculosis continues to be a major health problem in our country. Despite high prevalence of tuberculosis in women of child bearing age, only few cases of congenital tuberculosis have been reported [1], probably the frequency is under estimated as the clinical presentations are variable and a clinician may overlook the diagnosis unless one specifically looks for definite clues $[2,3]$. The importance of early diagnosis and treatment is crucial considering non specific nature of presentation and high mortality associated with it. We report two cases of congenital tuberculosis due to its rarity and highlight the importance of improved screening of women at risk.

\section{Case 1}

A 12 day old female neonate presented with fever, lethargy and poor feeding for past three days. She was born normally at term with normal Apgar scores to a 24 year old mother with a bad obstetrical history $\left(\mathrm{G}_{3} \mathrm{P}_{0} \mathrm{~A}_{2}\right)$. The current antenatal period was supervised at a primary health centre with an uneventful period. The birth weight was $2.3 \mathrm{~kg}$ and breast-feeding was established with no early neonatal problems but she had a weight loss of 500 gms. On admission she was lethargic and weighed $1.8 \mathrm{~kg}$. There was no respiratory distress, cyanosis or jaundice. Chest was clear with normal heart sounds. She had hepatosplenomegaly. Other systems were normal. Routine haematological investigations and liver function tests were normal. Initial chest radiograph showed clear lung fields and normal mediastinal shadows.

A clinical diagnosis of septicaemia was made and antibiotic therapy with ampicillin and gentamycin was started. There was no significant improvement and on sixteenth day of life she developed mild respiratory distress requiring oxygen supplement. A repeat radiograph revealed progressive bronchopneumonia (Fig. 1) and haematological test showed mild leucocytopenia. Ultrasound of the abdomen showed hepatosplenomegaly, portal adenopathy with no ascites.
Blood and urine cultures were sterile. Serological tests for toxoplasmosis, herpes, syphilis, cytomegalo virus and human immunodeficency virus were negative. Mantoux test was negative and so were gastric aspirates for acid fast bacilli (AFB) on three occasions. The antibiotics were changed to cefotaxime, amikacin and cloxacillin but she continued to deteriorate. Meanwhile the mother who was having high fever was diagnosed to have pulmonary tuberculosis. Her chest radiograph showed a thick wall cavity in right lung (Fig.2), positive mantoux test $(22 \mathrm{~mm})$ and submandibular tubercular lymphadenitis that was confirmed on fine needle aspiration

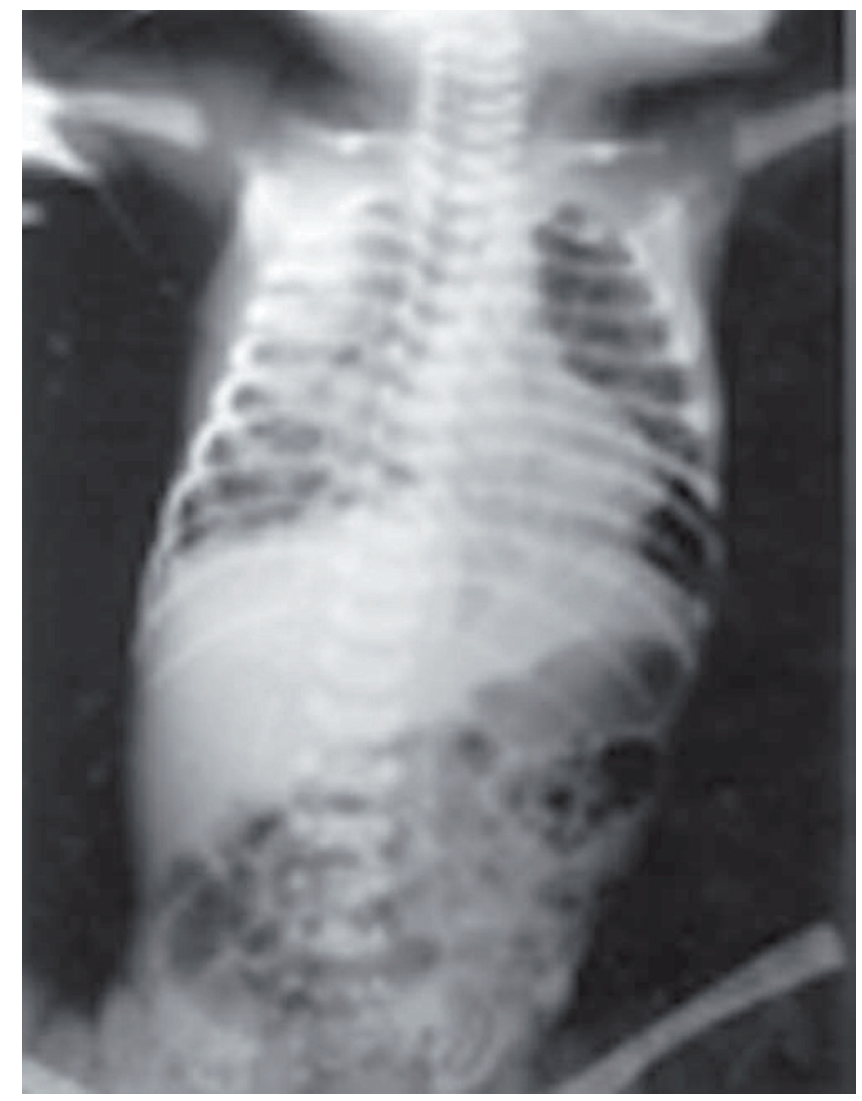

Fig. 1: Chest radiograph of neonate on $16^{\text {th }}$ day showing progressive bronchopneumonia

\footnotetext{
"Classified Specialist (Paediatrics), Military Hospital, Amritsar. ${ }^{+}$Dy Commandant, Command Hospital (WC), Chandimandir. ${ }^{*}$ ADMS, HQ Northern Command, 56 APO. ${ }^{* *}$ Classified Specialist (Pathology), Military Hospital, Ambala. ${ }^{++}$Ex-Classified Specialist (Radiodiagnosis), Military Hospital, Secunderabad.
}

Received : 05.09.2006; Accepted : 25.01.2007

Email : tomar15@rediffmail.com 


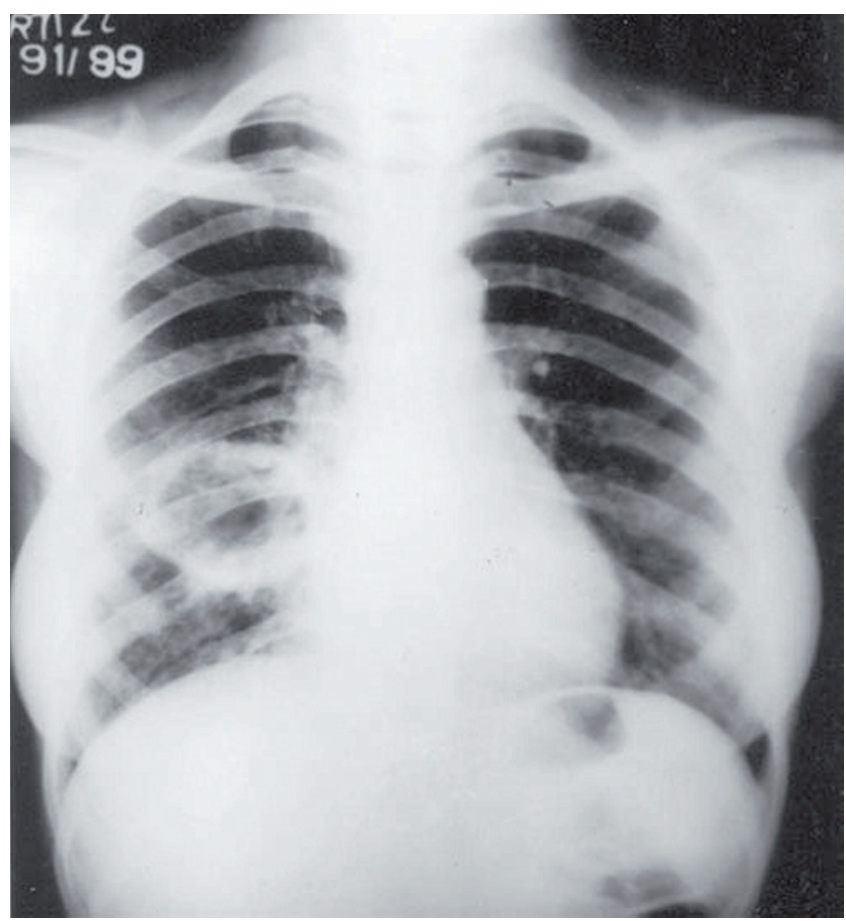

Fig. 2: Chest radiograph of mother revealing tubercular lung abscess (Right lung)

cytology (FNAC). Her endometrial biopsy showed typical tubercular granuloma confirming the source of infection and perinatal onset of this disease in the neonate. Isonex, rifampicin and pyrazinamide were started while amikacin was continued. There was good response with weight gain, increased feeding and respiratory distress regressed in a fortnight. The infant was followed up for two months on antitubercular drugs and the course was marked by good weight gain, regression of hepatosplenomegaly after which she was lost for follow up.

\section{Case 2}

A 39 day old female infant was admitted with a history of fever, cough, poor feeding and failure to gain weight since one month. She was born at term at home to a 24 year old primi mother. The infant was unimmunized and partially breast fed since birth, but on top milk since 20 days in view of her mother's admission for extensive tubercular bronchopneumonia. On examination, she was pale, lethargic, severely emaciated and weighed only $2.5 \mathrm{~kg}$. She had respiratory distress with chest recessions, bilateral crepitations and hepatosplenomegaly. Other systems were essentially normal. Investigations revealed haemoglobin of $8.2 \mathrm{gm} \%$, total leucocyte counts of $14,000 /$ cumm with $76 \%$ polymorphs and peripheral smear was suggestive of normocytic normochromic anaemia. Chest radiograph showed severe bronchopneumonic changes suggesting miliary mottling of both the lungs (Fig. 3). Mantoux test and gastric aspirates for AFB were negative. Cerebrospinal fluid showed four lymphocytes with normal sugar and proteins and a negative AFB smear. Ultrasound revealed hepatosplenomegaly with multiple hypoechoeic hepatic foci and enlarged portal lymphnodes. Guided liver biopsy was deferred due to her poor condition. Both mother and infant

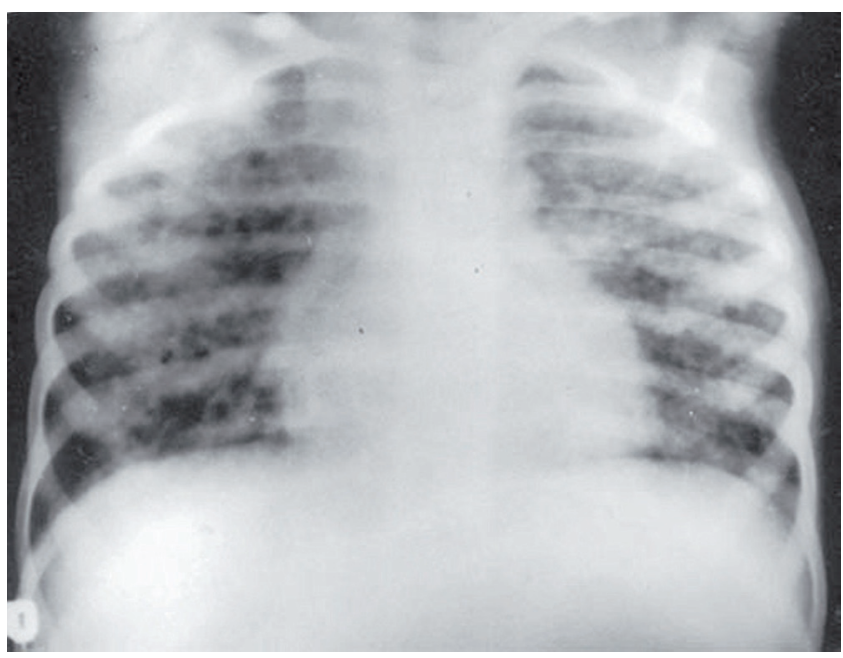

Fig. 3 : Radiograph suggesting miliary mottling of both the lungs

were anti-HIV antibody negative. A diagnosis of congenital tuberculosis was implied in view of the miliary mottling in chest radiograph with a positive close contact history and she was started on isonex, rifampicin, pyrazinamide and amikacin along with cefotaxime. She continued to deteriorate rapidly and additional management included steroids, inotropic support and blood transfusions. Nevertheless she succumbed to her condition on the sixth day of admission. A subcutaneous liver biopsy was done post-mortem as autopsy was refused by mother which confirmed the diagnosis showing tubercular granulomas with typical Langhans giant cells. Biopsy from endometrium of mother could not be done due to her deteriorating condition for which she was shifted to tertiary higher centre.

\section{Discussion}

Congenital tuberculosis is defined when tubercular infection is transmitted to the foetus either haematologically via the umbilical vein or by infected amniotic fluid, which is ingested or aspirated in utero, or during delivery [3]. It should be distinguished from acquired neonatal tuberculosis, where the infant is infected after birth by a contagious adult. Diagnostic criteria for congenital tuberculosis by Beitzke [4], included that tuberculosis should be bacteriologically proven and disease established in the first few days of life. The primary complex is in liver and if liver is not involved, then lesions are to be found within few days of birth or when extra uterine sources of infection can be eliminated with certainty. Since these were too rigid, Cantwell et al [5], proposed certain modifications in which the neonate should have a proven tubercular lesion with lesions in the first week of life, primary hepatic complex or caseating hepatic granuloma, tuberculosis infection of placenta or maternal genital tract or exclusion of postnatal transmission by thorough contact investigation [6]. Though some authors now suggest the term perinatal tuberculosis instead of congenital tuberculosis since distinguishing it from early postnatal acquired 
tuberculosis is a matter of epidemiological importance as they do not differ much with regard to presentation, treatment and prognosis [7].

Infection in such cases can be acquired through three different modes; transplacentally with primary complex in the liver, aspiration of infected amniotic fluid during passage through birth canal when lungs are primary focus and ingestion of infected material where the primary focus is in the gut [3]. An infant infected in utero will present with hepatic or more disseminated disease while those infected during birth may present with pulmonary or miliary disease. Pathologically, the striking fact about the tubercular lesion is the lack of host response due to which most infants do not develop tuberculin sensitivity throughout their illness. The first case qualifies for the diagnosis of congenital tuberculosis as tubercular infection of maternal genital tract was histopathologically confirmed while in second case the primary complex was histopathologically proven in liver. The lungs were also infected either due to dissemination of the disease or aspiration of the infected amniotic fluid, though continued postnatal infection could be completely excluded and hence fails on count of exclusion of postuterine infection by contact but we believe that the infection was most probably intrauterine as the usual interval of presentation in tuberculosis after exposure is about six weeks.

Congenital tuberculosis is particularly difficult to diagnose since the non-specific presenting signs and symptoms (because of lack of host response) such as respiratory distress, hepatosplenomegaly, jaundice, fever, lymphadenopathy, lethargy and failure to thrive are also seen in other nontubercular infections. In particular, common premature conditions such as chronic lung disease, patent ductus arteriosus (PDA), pneumonia and sepsis are readily mimicked by tuberculosis [6]. The clinical course is often fulminant and characterized by dissemination and meningitis with high (60\%) mortality [7]. Mantoux test is rarely positive and diagnosis is confirmed on basis of positive smear and/or culture results obtained from gastric washing, liver biopsy, lymphnode biopsy, spinal fluid, ear discharge or polymerase chain reaction studies [8]. Ultrasonography facilitates in early diagnosis and guided biopsies. Endometrial biopsy is useful in diagnosis of genital tuberculosis in the mother. Treatment should be started for two months with three drugs (isonex, pyrazinamide and rifampicin) and continued for next seven months (rifampicin and isonex), which could be longer with central nervous system involvement [9]. Hence diagnosis of tuberculosis in mother is very important. Diagnosis of tuberculosis in the mother of first case could be done retrospectively despite the chest radiograph showing a chronic lesion. Unlike our cases where diagnosis was quickly established in mothers of both cases, majority of mothers of infants with congenital tuberculosis might have no complaints $[1,8]$. However extra pulmonary tuberculosis in child bearing women is associated with a high incidence of infertility due to genital tuberculosis and is said to be one of the reasons for low incidence of congenital tuberculosis $[1,10]$.

\section{Conflicts of Interest}

None identified

\section{References}

1. Balasubramanian S, Shivram R, Padmasani LN, Nagaraju. Congenital tuberculosis. Indian J Pediatr 1999; 66:148-50.

2. Mathai J, Ravikumar VR, Ramaswamy M. Congenital tuberculosis. Under diagnosed or under reported ? Ind J Pediatrics 1994; 61:430-2.

3. Miller FJW. Tuberculosis in children. Evolution, Epidemiology, Treatment, Prevention, 1st ed. New Delhi: Churchill Livingstone, 1982; 220-4.

4. Beitzke H. Uber die andioborenee tuberculose infection. Erbebn Tuberk Forsh 1935; 7:1-12.

5. Cantwell M, Snider DE Jr, Cauthen GM, Onorato IM. Epidemiology of tuberculosis in the United States, 1985 through 1992. JAMA 1994; 272:535-9.

6. Mazade MA, Evans ME, Starke JR, Correa AG. Congenital tuberculosis presenting as sepsis syndrome: case report and review of the literature. Pediatr Infect Dis J 2001; 20:439-42.

7. Starke RS, Smith MH. Tuberculosis. In: Remington JS, Klein JO, editors. Infectious diseases of the foetus and newborn. Philadelphia: WB Saunders Company; 2001; 1184-7.

8. Hageman J, Shulamn S, Shreiber M, Lucks S, Yogev R. Congenital tuberculosis. Critical reappraisal of clinical findings and diagnostic procedures. Pediatrics 1980; 66:980-4.

9. Recommendations. Treatment of childhood tuberculosis: Consensus statement of IAP Working Group. Indian Pediatr 1997; 34:1093-6.

10. Jana N, Vasishta K, Saha S, Ghosh K. Obstetrical outcomes among women with extrapulmonary tuberculosis. N Engl J Med 1999; 341:645-9. 